\title{
Lipid profile variations in non alcoholic fatty liver disease
}

Original Research Article

\author{
Bharti Singla, Gesu Singla , Harsharan Kaur*
}

Department of Biochemistry, Adesh Institute of Medical Sciences and Research, Bathinda, India

Received: 05-08-2019 / Revised: 20-8-2019 / Accepted: 24-08-2019

\begin{abstract}
NAFLD is characterised by fatty infiltration of the liver, mostly in the form of triglycerides, which exceeds $5 \%$ of the liver weight. Insulin resistance impairs the suppression of lipolysis, and this leads to an increased release of free fatty acids from adipose tissue so that more are delivered to and taken up by the liver. This excess amount of free fatty acids can overload the hepatic mitochondrial beta oxidation system, the major pathway of fatty acid oxidation in the liver, leading to the accumulation of fatty acids in the liver. The present hospital-based, observational, analytical and comparative study was conducted on 80 subjects including 50 cases of ultrasonographically diagnosed NAFLD and 30 age and gender matched healthy subjects as controls. In this study, lipid profile was assessed in 50 USG diagnosed cases of NAFLD and 30 controls and the results were analysed statistically. The study found a statistically significant change in lipid levels in patients of Non alcoholic fatty liver disease indicating hepatic steatosis. In the present study, increased levels of cholesterol, triacylglycerides, LDL, VLDL and decreased levels of HDL were found. This study concluded that low fat diet, lifestyle modifications, exercises and yoga could be a potential tool to prevent the progression of NAFLD.
\end{abstract}

Key words: NAFLD, Cholesterol, LDL, Triacylglycerol, VLDL, HDL.

(C) The Author $(s) .2019$ Open Access. This work is licensed under a Creative Commons Attribution. The full terms of this license are available at our website and incorporate the Creative Commons Attribution. https://creativecommons.org/licenses/by/4.0/

\section{INTRODUCTION}

The liver plays a central role in lipid transport \& metabolism. A net change in the amount of lipid in the liver will occur if there is a change in the balance between the liver's uptake or synthesis of fatty acids and the liver's disposal of fatty acids by oxidation or export. [1,2] Non alcoholic fatty liver disease is a common hepatic disorder characterized by accumulation of fat in the liver parenchyma of the patients who do not drink excessive amount of alcohol. It is a clinicopathological syndrome characterized by wide spectrum of liver damage ranging from benign fatty infiltration to steatohepatitis, advanced fibrosis and cirrhosis.[3] Fatty liver, or hepatosteatosis, is characterized histologically by triglyceride accumulation within the cytoplasm of hepatocytes and refers to fat accumulation in the liver exceeding 5\%$10 \%$ by weight.[4]

*Corresponding Author

Harsharan Kaur

Department of Biochemistry, Adesh institute of medical sciences and research, Bathinda, Punjab, India. E-mail: sharan.sandhu35@gmail.com
Abnormalities in fatty acid metabolism, in conjunction with adipose tissue, hepatic, and systemic inflammation, are key factors involved in the development of insulin resistance, dyslipidemia, and other cardiometabolic risk factors associated with NAFLD.[5] A net retention of lipids within hepatocytes, mostly in the form of triglycerides, is a pre-requisite for the development of nonalcoholic fatty liver disease. The primary metabolic abnormalities leading to lipid accumulation is not well understood, but they could consist of alterations in the pathways of uptake, synthesis, degradation, or secretion in hepatic lipid metabolism resulting from insulin resistance.[6]

\section{MATERIALS AND METHODS}

The present study was conducted in Department of Biochemistry, Government Medical College, Patiala on 80 cases (including controls) reporting in Department of Medicine, Rajindra Hospital, Patiala from March, 2016 to November, 2016. The present hospital-based, observational, analytical and comparative study was subjected to statistically analysis. The study was approved by ethical committee of institute. 


\section{Sample Collection}

Specimen collection - Blood sample, one in plain vial was collected under all aseptic conditions by venipuncture. The plain vial sample is allowed to clot and the serum was then separated by ultracentrifugation of the sample at room temperature.

\section{Investigations}

1. Determination of Triglycerides (TG) GPO - PAP Method. [7]

Normal Range: $30-180 \mathrm{mg} \%$

2. Determination of Cholesterol: PAP Method. [8] Normal Range: $150-250 \mathrm{mg} \%$

3. Determination of HDL Cholesterol Phosphotungstic Acid Method[9]

Normal Range:

In males : $30-65 \mathrm{mg} / \mathrm{dl}$

In females : $35-80 \mathrm{mg} / \mathrm{dl}$

\section{Estimation of LDL-Cholesterol \& VLDL} Cholesterol

Formula [10,11,12]

VLDL-cholesterol $=\mathrm{TG} / 5$

LDL-cholesterol $=$ Total cholesterol $-(\mathrm{TG} / 5+\mathrm{HDL}-$ cholesterol)

These were estimated from the primary measurements by using the empirical equation of Friedewald et al.[10] after determining total cholesterol and triglycerides by Allain et al[11] (1974) and McGowan et al[12] (1983). Normal Range :

VLDL-cholesterol- 20-40 mg/dl

LDL-cholesterol- 114-153 mg/dl.

\section{RESULTS}

50 already diagnosed patients of NAFLD and 30 controls were taken and following observations were made from the study.

Table 1: Comparison of Serum Cholesterol Levels In NAFLD Patients and Control Group

\begin{tabular}{|l|l|l|l|l|}
\hline Parameter & Study Group & Mean \pm S.D $(\mathbf{m g} \%)$ & p value & Significance \\
\hline $\begin{array}{l}\text { S. Cholesterol } \\
\text { (N.V. 110-200 } \\
\text { mg\%) }\end{array}$ & Case & $244.92 \pm 18.96$ & \multirow{2}{*}{0.00} & HS \\
\cline { 2 - 3 } & Control & $184.20 \pm 8.43$ & & \\
\hline
\end{tabular}

The table above showed the comparison of $\mathrm{S}$. Cholesterol Levels in NAFLD patients and control group. Mean \pm S.D. of case group was $244.92 \pm 18.96$ $\mathrm{mg} \%$, while Mean \pm S.D. of control was $184.20 \pm 8.43$ $\mathrm{mg} \%$. The $\mathrm{p}$ value was 0.00 . So there was highly significant difference between the case and control groups on account of NAFLD.

Table-2: Comparison of Serum Triglycerides (Tg) Levels in NAFLD Patients and Control Group

\begin{tabular}{|l|l|l|l|l|}
\hline Parameter & Study Group & Mean \pm S.D $(\mathbf{m g} \%)$ & p value & Significance \\
\hline \multirow{2}{*}{$\begin{array}{l}\text { S. TG } \\
\text { (N.V.30-180 mg\%) }\end{array}$} & Case & $203.88 \pm 61.54$ & 0.00 & HS \\
\cline { 2 - 5 } & Control & $100.43 \pm 27.05$ & & \\
\hline
\end{tabular}

Above table showed the comparison of S. TG Levels in NAFLD patients and control group. Mean \pm S.D. of case group was $203.88 \pm 61.54 \mathrm{mg} \%$, while Mean \pm S.D. of control was $100.43 \pm 27.05 \mathrm{mg} \%$. The p value was 0.00 . So there was highly significant difference between the case and control groups on account of NAFLD.

Table-3: Comparison of Serum HDL Levels in NAFLD Patients and Control Group

\begin{tabular}{|l|l|l|l|l|}
\hline Parameter & Study Group & Mean \pm S.D $(\mathbf{m g} \%)$ & p value & Significance \\
\hline \multirow{2}{*}{$\begin{array}{l}\text { S. HDL } \\
\text { (N.V. 38-75 mg\%) }\end{array}$} & Case & $30.64 \pm 4.77$ & \multirow{2}{*}{0.00} & HS \\
\cline { 2 - 4 } & Control & $44.53 \pm 5.87$ & & \\
\hline
\end{tabular}


The table above showed the comparison of S. HDL Levels in NAFLD patients and control group. Mean \pm S.D. of case group was $30.64 \pm 4.77 \mathrm{mg} \%$, while Mean \pm S.D. of control was $44.53 \pm 5.87 \mathrm{mg} \%$. The p value was 0.00 . So there was highly significant difference between the case and control groups on account of NAFLD.

Table-4: Comparison of Serum LDL Levels in NAFLD Patients and Control Group

\begin{tabular}{|l|l|l|l|l|}
\hline Parameter & Study Group & Mean \pm S.D $(\mathbf{m g \%})$ & p value & Significance \\
\hline \multirow{2}{*}{$\begin{array}{l}\text { S. LDL } \\
\text { (N.V. } 114-153 \mathrm{mg} \%)\end{array}$} & Case & $175.76 \pm 19.69$ & & \\
\cline { 2 - 3 } & Control & $120.27 \pm 6.47$ & & HS \\
\hline
\end{tabular}

The table above showed the comparison of S. LDL Levels in NAFLD patients and control group. Mean \pm S.D. of case group was $175.76 \pm \mathbf{1 9 . 6 9} \mathbf{~ m g} \%$, while Mean $\mathbf{H}$ S.D. of control was $\mathbf{1 2 0 . 2 7} \pm \mathbf{6 . 4 7} \mathbf{m g} \%$. The p value was 0.00 . So there was highly significant difference between the case and control groups on account of NAFLD.

Table-5: Comparison of Serum VLDL Levels in NAFLD Patients and Control Group

\begin{tabular}{|l|l|l|l|l|}
\hline Parameter & Study Group & Mean \pm S.D $(\mathbf{m g} \%)$ & p value & Significance \\
\hline \multirow{2}{*}{$\begin{array}{l}\text { S. VLDL } \\
\text { (N.V. 20-40 } \mathrm{mg} \%)\end{array}$} & Case & $39.68 \pm 13.18$ & \multirow{2}{*}{0.00} & HS \\
\cline { 2 - 5 } & Control & $21.27 \pm 7.63$ & & \\
\hline
\end{tabular}

The table above showed the comparison of S. VLDL Levels in NAFLD patients and control group. Mean \pm S.D. of case group was $39.68 \pm 13.18 \mathrm{mg} \%$, while Mean \pm S.D. of control was $21.27 \pm 7.63 \mathrm{mg} \%$. The p value was 0.00 . So there was highly significant difference between the case and control groups on account of NAFLD.

\section{DISCUSSION}

The study found a statistically significant change in lipid levels in patients of Non alcoholic fatty liver disease indicating hepatic steatosis. In the present study, increased levels of cholesterol, triacylglycerides, LDL, VLDL and decreased levels of HDL were found. The present study resembled who found higher levels of S. Cholesterol and S. Triglycerides in NAFLD patients.[12-15] Gaharwar et al[14] 2015, also found lower levels of S. HDL and higher levels of S. LDL in NAFLD patients. The present study resembled Sharavnan et al[16] 2015, who found higher levels of S. VLDL in NAFLD patients. NAFLD was associated with increased SREBP-2 maturation, HMG CoA reductase (HMGCR) expression and decreased phosphorylation of HMGCR. Cholesterol synthesis was increased as measured by the circulating desmosterol:cholesterol ratio. miR-34a, a microRNA increased in NAFLD, inhibited sirtuin-1 with downstream dephosphorylation of AMP kinase and HMGCR.[17] The increased rates of fatty acid release into plasma and hepatic VLDL-TG secretion likely contribute to insulin resistance and hyper triglyceridemia that are commonly associated with
NAFLD.[18] Low serum concentration of HDL-C and higher concentrations of triglycerides, the key features of insulin resistance dyslipidemia, were significantly different between patients with and without NAFLD. Higher fasting triglyceride levels and LDL particle concentrations were also significant associates of NAFLD.[19]

\section{CONCLUSION}

As NAFLD is rapidly becoming an important problem. Undiagnosed, this condition may progress silently and result in cirrhosis, portal hypertension, and liver-related death in early adulthood. The assessment of lipid profile for liver injury makes the development of noninvasive, readily available, and easy-to-perform markers like Cholesterol, Triacylglycerides, HDL, VLDL, LDL a high priority for diagnostic purpose. So this study conveys the message that low fat diet, lifestyle modifications, exercises and yoga could be a potential tool to prevent the progression of NAFLD. 


\section{REFERENCES}

1. Marchesini G, Brizi M, Morselli-Labate AM. Association of nonalcoholic fatty liver disease with insulin resistance. Am Med J. 1999; 107: $450-55$.

2. Prasoon Kumar Saxena, Rosaline Mishra, Abhishek Bhardwaj, Moumita Barman, Rahul Chaudhary. A Conspectus: Hepatotoxicity Caused by Drug's. Indian J. Pharm. Biol. Res. 2018; 6(3):55-59.

3. Li Z, Clark J, Diehl AM. The liver in obesity and type 2 diabetes mellitus. Clin Liver Dis J. 2002; 6: 867-77.

4. Luxmi S, Sattar AR, Ara J. JLUHMS. 2008; 18893.

5. Obika M, Noguchi H. Diagnosis and Evaluation of Nonalcoholic Fatty Liver Disease Journal of Diabetes. 2012; 1-12.

6. Fabbrini E, Sullivan S, Klein S. Obesity and nonalcoholic fatty liver disease: biochemical, metabolic and clinical implications. Hepatology. 2010; 51: 679-89.

7. Mahaling UD, Basavaraj MM, Bika JA. Comparison of lipid profile in different grades of non-alcoholic fatty liver disease diagnosed on ultrasound Asian Pacific Journal of Tropical Biomedicine. 2013; 3(11): 907-12.

8. Burstein M, Schoinic HR, Morfin R. J. Lipid Res. 1970; 11(6): 583-95.

9. Rautela G.S, Leidtke R.J. Clin. Chem.1978;24(1):108-14.

10. Jimba $S$, Nakagami T, Takahashi M. Prevalence of nonalcoholic fatty liver disease and its association with impaired glucose metabolism in Japanese adults. Diabet Med J, 2005; 22: 1141-5.

11. Friedewald WT, Levy RJ, Fredrickson DS. Estimation of the concentration of low-density lipoprotein cholesterol in plasma without use of the preparative ultracentrifuge. Clin Chem 1972;18(6):499-502.

Conflict of Interest: None

Source of Support: Nil
12. Allain CC, Poon LS, Chan CS, Richmond W, Fu PC. Enzymatic determination of total serum cholesterol. Clin Chem 1974;20(4):470-5.

13. McGowan MW, Artiss JD, Strandbergh DR, Zak B. A peroxidise-coupled method for the colorimetric determination of serum triglycerides. Clin Chem. 1983;29(3):538-42.

14. Rao B, Kumar S, Rao S, Devi A. IJPBS. 2012; $2(2) ; 1-7$.

15. Gaharwar R, Trikha S, Margekar SL, Jatav OP, Ganga DP. Study of Clinical Profile of Patients of Non Alcoholic Fatty Liver Disease and its Association with Metabolic Syndrome. Journal of the association of physicians of India. 2015; 63: 12-16.

16. Nagaraj S, Kiran SS, Gandham R, Silvia WD, Nagaraja MR, Nasar A at al. Study of prevalence of non alcoholic fatty liver disease in type 2 diabetes mellitus patients and variations in liver function tests, lipid profile and mean platelet volume in patients with fatty liver in comparison with patients without fatty liver. IJRMS. 2016; 4(3): 871-6.

17. Sharavanan TK. Prevalence of non-alcoholic fatty liver disease in type 2 diabetes mellitus in a rural health care hospital. SJAMS. 2015; 3(5A): 18347.

18. Kerr TA, Davidson NO. Cholesterol and NAFLD: Renewed focus on an old villain. Hepatology. 2012; 56(5): 1995-8.

19. Wanless IR, Shiota K. The pathogenesis of nonalcoholic steatohepatitis and other fatty liver diseases: a four-step model including the role of lipid release and hepatic venular obstruction in the progression to cirrhosis. Semin Liver Dis 2004; 24: 99-106.

20. Trojak A, Miarka MW, Wozniakiewicz E, Malecki MT, Walus BII. Nonalcoholic fatty liver disease is associated with low HDL-Cholesterol \& Coronary Angioplasty in patients with type-2 diabetes. Med Sci Monit. 2013; 19: 1167-72. 